\title{
Process of soil destruction: experimental results
}

\author{
Irina Troyanovskaya ${ }^{1,2, *}$, Olga Grebenshchikova ${ }^{1,3}$, and Ivan Zhitenko ${ }^{1}$ \\ ${ }^{1}$ South Ural State Agrarian University, Applied Mechanics Department, Chelyabinsk, Russia 454080 \\ ${ }^{2}$ South Ural State University, Car and Caterpillar Vehicles Department, Chelyabinsk, Russia 454080 \\ ${ }^{3}$ Air Force Military Educational and Scientific Centre Air Force Academy, Gorodok-11, Chelyabinsk, \\ Russia, 454015
}

\begin{abstract}
The article presents the results of experimental studies of the ripping resistance force on a tracked bulldozer-ripper manufactured by the Chelyabinsk Tractor Plant. The experiment covers three ripping depths and eight angles. It has been experimentally established that the dependence of the ripping resistance force on each of these parameters is quadratic. The authors propose the use of a complex parameter which is equal to the product of the ripping depth by the angle when studying the ripping process. The use of the complex parameter for two-factor analysis allowed them to reduce the degree of the studied dependence while preserving the required accuracy. The complex parameter reflects the relationship between the design parameters of the ripper tooth, the ripping depth, and angle.
\end{abstract}

\section{Introduction}

Ripping is an integral part of the technological cycle both for agricultural and road building machines $[1,2]$. The scheme and parameters of the working tool and the hinged system mainly determine the soil cultivation nature $[3,4]$. Two main types are identified among the variety of hinged systems: those with one-sided (Fig. 1a) and two-sided (Fig. 1b) force closure.

Systems with one-sided force closure do not provide positive penetration of the working tool into the soil or ensure its free movement relative to the tractor. Such systems are typical for agricultural tillage machines. Their theory is well-developed today [5, 6].

The hinged systems of road building machines designed for the destruction of firm, frozen, and rocky soils are fundamentally different from the hinged systems of ripping machines. Their hinged systems ensure the positive penetration of the working tool into the soil. The issues of ripping process optimization require additional studies.

It has been established that the forces acting on the working tool in relation to the soil depend on the ripping process parameters: ripping depth $\mathrm{h}$ and the angle $\alpha$. At present, these dependences are mainly studied experimentally $[7,8]$. Theoretical studies are reduced mainly to the models of a discrete element $[9,10,11]$.

\footnotetext{
*Corresponding author: tripav63@mail.ru
} 

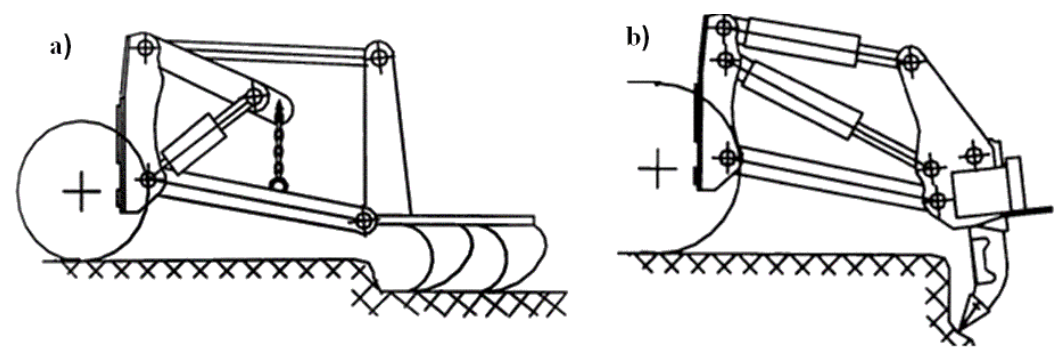

Fig. 1. Hinged systems with one-sided (a) and two-sided (b) force closure.

\section{Methods of the experimental studies}

Experimental studies were conducted using a tracked bulldozer ripper. Its controls allow for control of ripping depth $\mathrm{h}$ and angle $\alpha$. Loam of medium density (in summer) and frozen loam (in winter) was utilized for these studies. The experiment was completed at various ripping angles $\alpha=38^{0}, 43^{\circ}, 45^{\circ}, 47^{0}, 50^{\circ}, 53^{0}, 55^{\circ}, 60^{\circ}, 65^{\circ}$ and depth $(h \approx 0.7 \mathrm{~m} ; h \approx 0.9 \mathrm{~m}$; $h \approx 1.1 \mathrm{~m}$ ). The depth h was measured every $0.1 \mathrm{~m}$ of the route.

The objective of the experiment was to determine traction resistance (horizontal component $P_{x}$ ) and penetration resistance (vertical component $P_{y}$ ) at different ripping parameters. Tension sensors were stuck to the ripper to measure the resistance forces.

By attaching tension sensors, we were able to measure the forces perpendicular to the front section $P_{K}$ and on the foot $P_{N}$ of the ripper (Fig. 2).

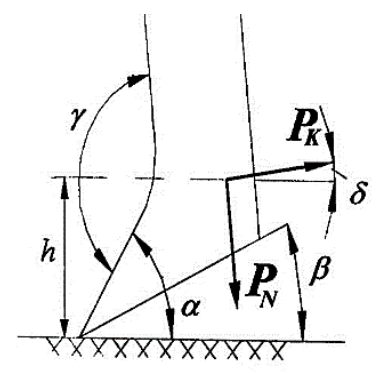

Fig. 2. Scheme for the determination of the components of the force of ripping resistance.

Based on the measured forces of $P_{K}$ and $P_{N}$, we calculated the desired components of the force of ripping resistance of $P_{x}$ and $P_{y}$ :

$$
\begin{gathered}
P_{x}=P_{K} \cos \delta+P_{N} \sin \delta \\
P_{y}=-P_{K} \sin \delta+P_{N} \cos \delta
\end{gathered}
$$

where $\delta=0,5 \pi+\alpha-\gamma$ is slope angle of the ripper shank to the vertical, $\beta$ is the slope angle of the ripper foot to the horizon, $\alpha$ is the ripping angle, and $\gamma$ is the positive angle of the ripper shank.

\section{Experiment results}

Resistance forces were measured at constant values of ripping depth $\mathrm{h}$ or angle $\alpha$. All the experimental dependences were approximated using the least square method. 
The appearance of the approximating dependencies (Fig. 3) demonstrated the non-linear nature of the relationship between traction resistance force and ripping depth $P_{x}(h)$ and the angle $P_{x}(\alpha)$.
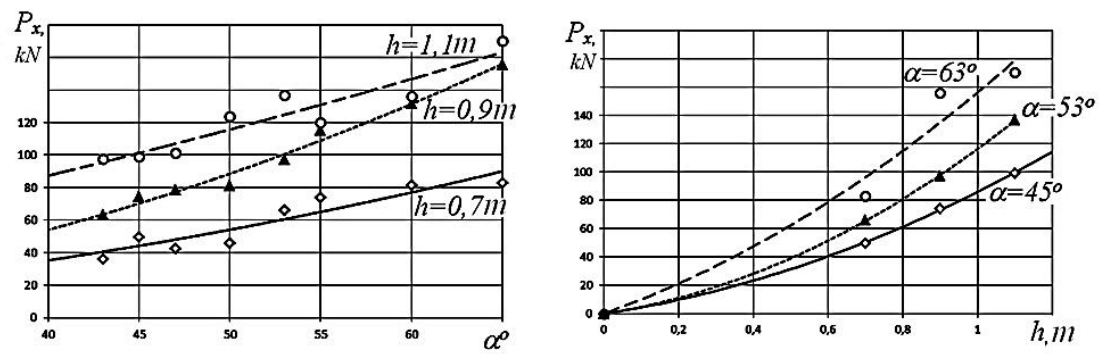

Fig. 3. Dependencies of traction resistance force $P_{x}$ on ripping angle $\alpha(a)$ and ripping depth $h(b)$.

For example, the dependencies presented in Figure 3 are as follows:

$$
\begin{aligned}
& \text { for } h=0,7 \mathrm{~m} \quad P_{X}(\alpha)=0,0197 \alpha^{2}+0,0979 \alpha, \\
& \text { for } h=0,9 m \quad P_{X}(\alpha)=0,0383 \alpha^{2}+0,115 \alpha, \\
& \text { for } h=1,1 m \quad P_{X}(\alpha)=0,0056 \alpha^{2}+2,0895 \alpha, \\
& \text { for } \alpha=45^{0} P_{X}(h)=0,0071 h^{2}+0,2175 h, \\
& \text { for } \alpha=53^{0} P_{X}(h)=0,0098 h^{2}+0,3979 h, \\
& \text { for } \alpha=65^{0} P_{X}(\alpha)=0,0043 h^{2}+1,2015 h .
\end{aligned}
$$

Analogous qualitative dependencies of $P_{y}(h)$ and $P_{y}(\alpha)$ are also observed for the vertical component of the resistance force.

\section{Experimental data processing}

When processing the experimental data, it was suggested to simultaneously use the dependence on the two parameters $(\alpha$ and $h$ ). However, the use of a quadratic form of

$$
P(\alpha, h)=b_{1} \alpha^{2}+b_{2} h^{2}+b_{3} \alpha h+b_{4} \alpha+b_{5} h+b_{6},
$$

where $b_{1}, b_{2} \cdot b_{3}, b_{4}, b_{5}, b_{6}$ are empirical coefficients, does not lead to a good result because recording one of the parameters, for example $h$, and obtaining good approximations in the range of large values of $\alpha$ and $h$, does not correspond to the physical phenomenon at small values of these parameters. The function $P(\alpha, h)$ should give a rather close approximation in the range of values of the conducted experiment and meet the physical meaning in the range of extrapolation [12]. The following fourth-order polynomial meets these requirements:

$$
P(\alpha, h)=b_{1} \alpha h+b_{2}(\alpha h)^{2} .
$$

In addition, function (3) is characterized by a smaller number of empirical coefficients bi, than by formula (2). The results of processing the experiment using formula (3) are shown in Fig. 4. 


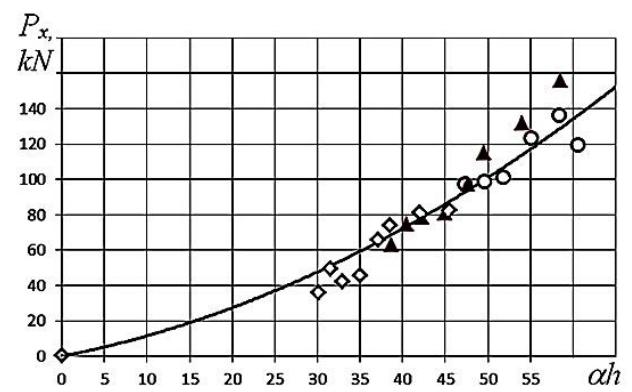

Fig. 4. The dependence of the force of traction resistance $P_{x}$ from the complex parameter $\alpha h$ : $P_{x}=2.10-6 \alpha h+0,0092(\alpha h)$

The results of experimental processing using formula (3) coincide with a sufficient degree of adequacy with theoretical studies dealing with the digging process [13, 14].

\section{Conclusions}

We experimentally established that the forces acting on the working part of the ripper are equally dependent on the ripping depth $\mathrm{h}$ [15] and the angle $\alpha$ [16].

Traction resistance $P_{x}$ and penetration resistance $P_{y}$ have a quadratic dependence on the ripping depth $h$ and the angle $\alpha$.

Using the complex parameter $\alpha$ h when describing the dependencies of $P_{x}(\alpha h)$ and $P_{y}(\alpha h)$ allows us to reduce the degree of the fourth-order polynomial to the second-order polynomial while preserving the required accuracy.

In addition, the complex parameter $\alpha h$ allows us to obtain a single dependence for each type of soil and reflects the relationship between the design parameters of the ripper tooth, ripping depth, and angle.

Our approach makes it possible to set the task of optimizing the ripping process depending on the type of the excavated soil [17].

\section{References}

1. M. Smith, L. Collis, P.G. Fookes. Aggregates: Sand, Gravel and Crushed Rock Aggregates for Construction Purposes (Bath: The Geological Society of London 2001)

2. I. Dyakov, V. Ivkin, A. Popovich. A Soil Loosening Machine for Winter Earth-Moving in Transport Construction, Transport, vol. 22(4), pp. 316-319 (2007).

3. E. McKyes, J. Maswaure, Effect of Design Parameters of Flat Tillage Tools on Loosening of a Clay Soil, Soil \& Tillage Research, vol. 43(3-4), pp. 195-204 (1997).

4. V.V Blednykh, P.G. Svechnikov, I.P. Troyanovskaya, Tractor Plough with Repeated Cutting Angle on Working Elements, Procedia Engineering, vol. 206, pp. 1577-1582 (2017)

5. P.P. Raj, Soil Mechanics \& Foundation Engineering (Chennai: Pearson Education India 2008)

6. V.V Blednykh, P.G Svechnikov, I.P. Troyanovskaya, Moldboard surface universalization of the ploughshare operating unit, Procedia Engineering, vol. 129, pp. 6974 (2015)

7. S.V. Kravets, O.V. Stinio, The determination of the force of blocked cuting of soil and coefficient of energy intensity of deep ripping of soil, Naukovyi Visnyk Natsionalnoho Hirnychoho Universytetu, vol. 3, pp. 24-28 (2016) 
8. T. Yoneyama, Y. Hatamura, T. Nagao, K. Ogaki, Y. Iwamoto, Measurement of stress and temperature on the ripper point surface in the ripping of rock ground, Transactions of the Japan Society of Mechanical Engineers, Part C, vol. 63(613), pp. 3313-3319 (1997) 9. I. Shmulevich, Z. Asaf, D. Rubinstein, Interaction between soil and a wide cutting blade using the discrete element method, Soil and Tillage Research, vol. 97(1), pp. 37-50 (2007) 10. Z. Asaf, D. Rubinstein, I. Shmulevich, Determination of discrete element model parameters required for soil tillage, Soil and Tillage Research, vol. 92(1-2), pp. 227-242 (2007)

11. H.H. Basarir, C.C. Karpuz, L.L. Tutluoğlu. 3D modeling of ripping process, International Journal of Geomechanics, vol. 8(1), pp. 11-19 (2008)

12. L. Pelevin, I. Gorbatyuk, S. Zaichenko, V. Shalenko, Developing a mathematical substantiation for the physical modelling of the soil-ripping equipment work process, Eastern European Journal of Enterprise Technologies, vol. 6(2-90), pp. 52-60 (2017)

13. W.C. Swick, J.V. Perumpral, A model for predicting soil-tool interaction, Journal of Terramechanics, vol. 25 (1), pp. 43-56 (1988)

14. Z. Decha, Y. Yusu, A dynamic model for soil cutting by blade and tine, Journal of Terramechanics, vol. 29 (3), pp. 317-327 (1992)

15. S.A. Al-Suhaibani, A.E. Ghaly, Effect of plowing depth of tillage and forward speed on the performance of a medium size chisel plow operating in a sandy soil, American Journal of Agricultural and Biological Science, vol. 5(3), pp. 247-255 (2010)

[16] J. Tong, B.Z. Moayad, Effects of rake angle of chisel plough on soil cutting factors and power requirements: A computer simulation, Soil and Tillage Research, vol. 88(1-2), pp. 55-64 (2006)

17. V.V. Blednykh, P.G. Svechnikov, I.P. Troyanovskaya, Moldboard surface universalization of the ploughshare operating unit, Procedia Engineering, vol. 150, pp. 1297-1302 (2016) 